\title{
MASSMOMENTSOF INERTIA OF JOINED WING UNMANNED AERIAL VEHICLE
}

\author{
DimoZafirov \\ Associate professor, TATT, TechnicalUniversity-Sofia, branchPlovdiv, Bulgaria,zafirov@tu-plovdiv.bg
}

\begin{abstract}
Determination of mass moments of inertia of aircraft during the early stages of design is of great importance for the sizing of control surfaces and the evaluation of the dynamic characteristics. In the article are reviewed the problems of the analytic calculation of mass moments of inertia of an unmanned air vehicle at the conceptual design stage, of their automated determination with a CAD/CAMcodeatthedetaileddesignstageandoftheirmeasurementafterexperimentalmodelsareproduced.A comparison is made of the accuracy of the different methods.
\end{abstract}

Keywords: UAV, momentsofinertia, physicalpendulum, torsion pendulum.

\section{INTRODUCTION}

In order aircraft to have high maneuverability, acceptable stability and control and to alleviate gust loads, they should have low mass moments of inertia about the body-axis coordinate system $[1,2,3,4,5,6]$.The values of the moments increase by the fifth order of the increase of the size of the aircraft. At the same time their sizes constantly increase because of considerations for economic efficiency and reduction of environmental foot print.

The determination of mass moments of inertia in the design process of aircraft is a difficult problem. At the early stages of design, we do not posses the necessary information even for their approximate evaluation. We need their values at this stage for choice of an optimal variant, determination of control and stability performance of the aircraft being designed; determination of the requirements for the control system and the autopilot. Because of the high degree of uncertainty, big number of elements, their complex shape and many variants under review, it is extremely difficult to establish with the necessary fidelity the values of mass moments of inertia at this moment. At these stages, analytical or table methods that require a lot of effort to calculate, are used. For the unmanned air vehicles (UAV), the choice of optimal configuration is dependent to a great extent on the minimization of inertial moments.

With the contemporary design methods, at the stages of preliminary and detailed design, anaccurate 3D model of the aircraft is created, from which we can obtain the values of the inertial moments with greater precision than with theanalyticalortablemethods, but it is still not sufficient for the processes of optimization and integration.
At the finishing stages of design, we usually posses experimental models that we can use to measure the moments of inertia. This can be accomplished on experimental stands on earth or during flight tests. Because of the great dimensions and mass of aircraft, very big stands are required, and with flight test the accuracy is not high enough.

As for the UAVs, most do not have large dimensions and it is possible to measure their inertial moments on experimental stands with the required accuracy.

\section{EQUATIONS OF MOTION OF UAV}

In the classical mechanics, utilizing the principle of super position the motion of a body is represented as constituting from a translational and a rotational motion [7].

The translational motion is described by Newton's second law:

$$
\vec{F}=m \vec{a},
$$

And the rotational motion with the Euler's equation:

$$
\overrightarrow{\mathrm{M}}=I \vec{\alpha}
$$

Here $\boldsymbol{I}$ is an inertia matrix, which for the rotational motions performs role similar to mass for translational motion and for that reason is sometimes referred to as angular mass.

$$
I=\left[\begin{array}{ccc}
I_{x x} & -I_{x y} & -I_{x z} \\
-I_{y x} & I_{y y} & -I_{y z} \\
-I_{z x} & -I_{z x} & I_{z z}
\end{array}\right]
$$


In order to create the model for the rotational motion of the UAV, the values of moments of inertia about body-axis coordinate system with an origin at the center of mass of the aircraft are needed.

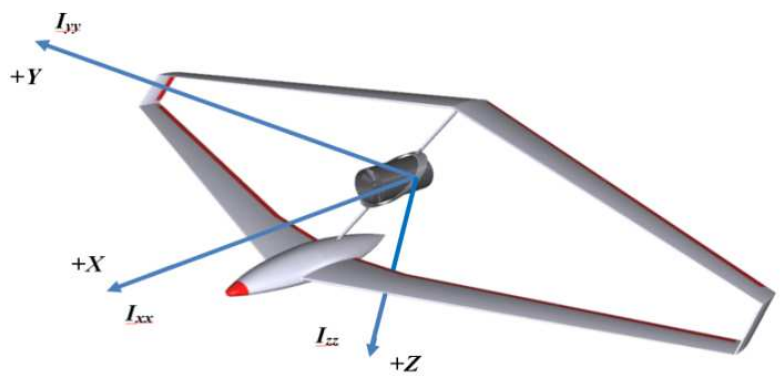

Fig. 1.Body-axis coordinate system with an originat the center of mass of the aircraft and inertial moments

Therotationalmotionaboutbody-axis coordinate system of the aircraftis presented as:

$$
\begin{array}{r}
I_{x x} \ddot{\varphi}=M_{x x} ; \\
I_{y y} \ddot{\theta}=M_{y y} ; \\
I_{z z} \ddot{\psi}=M_{z z} .
\end{array}
$$

The analysis of the equations reveals that as the values of inertial moments are reduced the aircraft will posses higher maneuverability and agility with constant control moments, or for required maneuverability and agility there will be needed smaller control moments, smaller control planes and/or control efforts.

\section{MASS MOMENTS OF INERTIA OF AIRCRAFT}

From the theoretical mechanics it is known that the moment of inertia of a solid body rotating about a specified axis is calculated by the following integral:

$$
\mathrm{I}_{\mathrm{ii}}=\int_{\mathrm{V}} \rho(\mathrm{r}) \mathrm{d}(\mathrm{r})^{2} \mathrm{dV}(\mathrm{r}) \text {. }
$$

The aircraft is made up of a great number of parts with different density, as well as large hollow parts filled with air. When flying at different heights the density of the air varies and so does the value of the inertial moment. Besides that it depends on the changes in the mass of the pay load and its position, as well as the remaining quantity of fuel and its distribution in the tanks.

During the conceptual design we can make the assumption that the variants that meet the requirements to the aircraft at maximum take off mass will be satisfactory at other flight conditions.
At this early stage of design we have very little information about the masses of the parts and their position in space, so it is appropriate to use approximate analytical dependencies and statistical data for the determination of moments of inertia for each variant. At the preliminary design stage it is appropriate to use for the calculation of the moments of inertia the 3D CAD model of the base variant. At the detailed design stage except with the full 3DCAD model the moments of inertia are determined experimentally through laboratory and flight tests.

In [7] is described the analytical determination of moments of inertia at the conceptual and preliminary design stages. At the detailed design stage with the availability of a full 3DCAD model the moments of inertia are determined automatically by the software with a built-in numerical code. After an experimental model is made the moments might be verified through laboratory tests.

\section{METHODS FOR THE DETERMINATION OF} MASS MOMENTS OF INERTIA

\subsection{Analytical Methods}

During the conceptual design of aircraft with a joined wing approximate values for the masses of the basic elements and their disposition are assumed for each variant being reviewed. The moment of inertia about $\boldsymbol{x}$ axis of the body-axis coordinate system is calculated using the formulae based on HuygensSteiner'sparallel axis theorem:

$$
\begin{aligned}
& I_{x x}=\sum_{j=1}^{n} I_{x_{j}} \\
& I_{x x_{j}}=I_{e l_{x x_{j}}}+m_{j} r_{j}^{2} .
\end{aligned}
$$

In an analogous way are calculated the moments of inertia about the other axis of the coordinate system. At fig. 2 an example break-up of an UAV to elements for the determination of inertial moments during the conceptual design is shown and in Table are given the values of moments of inertia of the elements of a particular variant of an UAV.

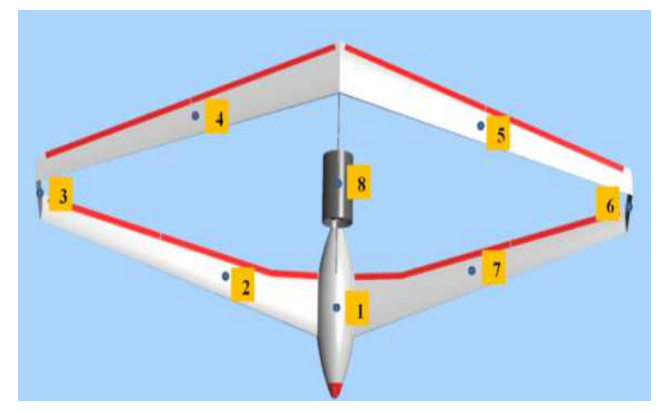

Fig 2 Elements of joined wing aircraft for analytical calculations of moments of inertia 
The results from analytical calculations of moments of inertia are given in table 1 and table 2 .

\section{Table 1}

\begin{tabular}{|c|c|c|c|c|c|c|c|c|c|c|}
\hline \multirow[t]{2}{*}{ No. } & $\mathrm{X}$ & $\mathbf{Y}$ & Z & $r_{x x}$ & $r_{y y}$ & $\mathbf{r}_{z z}$ & m & m.r..$_{x x}{ }^{2}$ & $\mathbf{m} \cdot \mathbf{r}_{\mathrm{yy}}{ }^{2}$ & $\mathbf{m} \cdot \mathbf{r}_{z z}{ }^{2}$ \\
\hline & $\mathbf{m}$ & m & m & m & m & $\mathbf{m}$ & $\mathrm{kg}$ & kg. $\mathrm{m}^{2}$ & kg. $\mathrm{m}^{2}$ & kg. $\mathrm{m}^{2}$ \\
\hline 1 & 0,106 & 0 & 0,142 & 0,142 & 0,1772 & 0,106 & 3,712 & 0,07485 & 0,11656 & 0,04171 \\
\hline 2 & 0,102 & 0,443 & 0,078 & 0,44981 & 0,12841 & 0,45459 & 0,41 & 0,08296 & 0,00676 & 0,08473 \\
\hline 3 & $-0,08$ & 0,9 & $-0,29$ & 0,94557 & 0,30083 & 0,90355 & 0,032 & 0,02861 & 0,0029 & 0,02612 \\
\hline 4 & $-0,702$ & 0,443 & $-0,418$ & 0,60908 & 0,81702 & 0,83009 & 0,41 & 0,1521 & 0,27369 & 0,28251 \\
\hline 5 & $-0,702$ & $-0,443$ & $-0,418$ & 0,60908 & 0,81702 & 0,83009 & 0,41 & 0,1521 & 0,27369 & 0,28251 \\
\hline 6 & $-0,08$ & $-0,9$ & $-0,29$ & 0,94557 & 0,30083 & 0,90355 & 0,032 & 0,02861 & 0,0029 & 0,02612 \\
\hline 7 & 0,102 & $-0,443$ & 0,078 & 0,44981 & 0,12841 & 0,45459 & 0,41 & 0,08296 & 0,00676 & 0,08473 \\
\hline \multirow[t]{2}{*}{8} & $-0,075$ & 0 & $-0,065$ & 0,065 & 0,09925 & 0,075 & 1,56 & 0,00659 & 0,01537 & 0,00878 \\
\hline & & & & & & Sum & 6,976 & 0,60877 & 0,69861 & 0,83721 \\
\hline
\end{tabular}

\section{Table 2}

\begin{tabular}{|c|c|c|c|c|c|c|c|c|c|}
\hline No. & $\mathbf{I}_{\text {elxx }}$ & $\mathbf{I}_{\text {elyy }}$ & $\mathbf{I}_{\text {elzz }}$ & $\mathbf{m} \cdot \mathbf{r}_{\mathrm{xx}}{ }^{2}$ & $m \cdot r_{y y}{ }^{2}$ & $\mathbf{m} \mathbf{r z}_{\mathrm{zz}}{ }^{2}$ & $I_{x x}$ & $\mathbf{I}_{\mathrm{yy}}$ & $I_{z z}$ \\
\hline & kg.m ${ }^{2}$ & kg.m $\mathrm{m}^{2}$ & kg. $\mathrm{m}^{2}$ & kg. $\mathrm{m}^{2}$ & kg.m $\mathrm{m}^{2}$ & kg.m ${ }^{2}$ & kg. $\mathrm{m}^{2}$ & kg.m ${ }^{2}$ & kg.m ${ }^{2}$ \\
\hline 1 & 0,216 & 0,24 & 0,142 & 0,07485 & 0,11656 & 0,04171 & 0,29085 & 0,35656 & 0,18371 \\
\hline 2 & 0,102 & 0,083 & 0,078 & 0,08296 & 0,00676 & 0,08473 & 0,18496 & 0,08976 & 0,16273 \\
\hline 3 & 0,08 & 0,01 & 0,02 & 0,02861 & 0,0029 & 0,02612 & 0,10861 & 0,0129 & 0,04612 \\
\hline 4 & 0,102 & 0,083 & 0,118 & 0,1521 & 0,27369 & 0,28251 & 0,2541 & 0,35669 & 0,40051 \\
\hline 5 & 0,102 & 0,083 & 0,118 & 0,1521 & 0,27369 & 0,28251 & 0,2541 & 0,35669 & 0,40051 \\
\hline 6 & 0,08 & 0,01 & 0,02 & 0,02861 & 0,0029 & 0,02612 & 0,10861 & 0,0129 & 0,04612 \\
\hline 7 & 0,102 & 0,083 & 0,078 & 0,08296 & 0,00676 & 0,08473 & 0,18496 & 0,08976 & 0,16273 \\
\hline 8 & 0,075 & 0,163 & 0,065 & 0,00659 & 0,01537 & 0,00878 & 0,08159 & 0,17837 & 0,07378 \\
\hline \multicolumn{7}{|c|}{ Moment of inetria of UAV } & 1,46777 & 1,45361 & 1,47621 \\
\hline
\end{tabular}

\subsection{Numerical Methods}

At the detailed design stage when a CAD/CAM system is used a full three dimensional model of the aircraft is created. This model contains information about the volumes and densities of all elements and their position which allows by numerical integration of (5) and applying the Huygens-Steiner's parallel axis theorem to calculate the inertial moments of UAV with great precision. In this case, in order to achieve higher accuracy, it is necessary the density of hollow volumes to equal that of the air.

\subsection{Experimental Methods}

Laboratory methods for experimental determination of mass moments of inertia of aircraft are based on the pendulum principle. The aircraft is oscillating about an axis that is not passing through its center of mass and is parallel to the axis about which we want to measure the moment of inertia. The mathematical model of a pendulum for small-angle oscillations in vacuum (without air resistance) is described with the following equation [8]:

$$
I \frac{d^{2} \theta}{d t^{2}}+b \theta=0
$$

Solvingthisequationweobtaintheperiodofoscillationandthemom entofinertia:

$$
b=m g l
$$

$$
\begin{aligned}
& T=\frac{2 \pi}{\sqrt{b / l}} \\
& I=\frac{T^{2} b}{4 \pi^{2}}=\frac{T^{2} m g l}{4 \pi^{2}} \\
& I_{C . G . .}=\frac{T^{2} m g l}{4 \pi^{2}}-m l^{2}
\end{aligned}
$$

The mass of the object under examination is determined by weighting. The weight $\boldsymbol{W}$, measured in a laboratory with spring scales gives the net weight of the object. An object with mass $\boldsymbol{m}$ and volume $\boldsymbol{V}$, immersed in the atmosphere, is acted upon by two forces - gravity and aerostatic [7]:

$$
m=\frac{w}{g}+\rho V
$$

In order to determine the mass of the object besides the weight we need to know its volume, the air density (or air pressure and temperature) at the moment of the measurement and the gravity acceleration.At the early stages of design we can supply only the approximate volume, which we can define more accurately at each of the next stages.

While moving through the air the oscillating parts drag with them a volume of air and this can be accounted for by adding the mass of the air that is carried with them to their mass. Thisadditionalmasswill depend on the resistance the body faces while moving through the air. If the density of the measured body is high, this additional mass is fairly small and might be ignored, but this is not the case with aircraft.The value of this mass depends on the wings aspect ratio. A more detailed description how to calculate this mass is given in [8].Then:

$$
m_{e f f}=m+m_{a}=\frac{w}{g}+\rho V+m_{a}
$$

Where $m_{e f f}$ is the aircraft's mass and is the additional mass, corresponding to the influence of the dragged air. In practice the motion of the pendulum will be slowed by the friction, which is not accounted for in the idealized case. The experimentally measured period of oscillations will be longer than the one calculated by the above equations. If the decrease in the amplitude of the oscillations is less than $1 / 10$ of the initial amplitude, the error due the environmental resistance will be below $0,02 \%$ and might not be accounted for. Equations (10) and (11), describing the motion of the pendulum in vacuum are applicable for pendulum oscillating in air, but in this case $\boldsymbol{I}$ and $\boldsymbol{m}$ refer to virtual values of the moment of inertia and mass of pendulum. The differences between these values are due to the following phenomena: flotation of the structure, the entrapped air and the effect of added mass. 
The real mass of the structure of a pendulum oscillating in the atmosphere will be:

$$
m_{s}=\frac{W}{g}+V_{s} \rho,
$$

The real mass of the structure, together with the entrapped air, will be:

$$
m=\frac{W}{g}+V_{s} \rho+\left(V-V_{s}\right) \rho
$$

Or

$$
m=\frac{W}{g}+V \rho .
$$

The object drags some of the surrounding air with its motion. In [9] it is accepted, that the influence of the dragged air might be accounted for by the addition of a complementary moment of inertia to the moment of inertia of the object:

$$
I_{A}+m_{A} L^{2}
$$

Where $\boldsymbol{I}_{A}$ the complementary moment of inertia about the mass is center and $\boldsymbol{m}_{\boldsymbol{A}}$ is the complementary mass. For physical pendulum, where $\boldsymbol{I}_{\boldsymbol{V}}$ is the virtual moment of inertia about the center of mass:

$$
I_{V}=I_{S}+I_{E}+I_{A}=\frac{T^{2} m l}{4 \pi^{2}}-\left(m+V \rho+m_{A}\right) l^{2}
$$

The unknowns in (19) are $\boldsymbol{I}_{\boldsymbol{V}}$ and $\left(\boldsymbol{V} \boldsymbol{\rho}+\boldsymbol{m}_{\boldsymbol{A}}\right)$ could be determined through swinging at two different lengths of the pendulum.

The real moment of inertia $\boldsymbol{I}_{i i}$ about an axis, passing through its center of mass, will be composed of four elements. The first one will be $\boldsymbol{I}_{s}$, representing the moment of inertia of the structure. These condone will be $\boldsymbol{I}_{E}$, representing the moment of inertia of the entrapped air the third one will be $\boldsymbol{I}_{\boldsymbol{A}}$, representing the moment of inertia of the adjoined mass air. The fourth one will be $\boldsymbol{I}_{\boldsymbol{G}}$, representing the moment of inertia of the swimming gear [8]:

$$
\begin{gathered}
I_{i i}=\frac{T^{2} m g l}{4 \pi^{2}}=I_{S}+I_{E}+I_{A}+I_{G} \\
I_{G}=\frac{T^{\prime 2} m^{\prime} l^{\prime}}{4 \pi^{2}}
\end{gathered}
$$

\subsubsection{Physical Pendulum Method}

For the physical pendulum the pivot axis is horizontal and passes through the suspension points, but not through the pendulum's center of mass. The characteristic constant for this pendulum and its moment of inertia will be as follows:

$$
b=m g l
$$

$$
I=\frac{T^{2} m g l}{4 \pi^{2}}
$$

For the physical pendulum the moment of inertia about an axis passing through the center of mass is expressed by the equation:

$$
I=\frac{T^{2} m g l}{4 \pi^{2}}-m l^{2}
$$

\subsubsection{Torsion Pendulum Method}

For bifilar Torsional pendulum with twin threads the axis of oscillation is vertical, passes through the center of mass of the system between the threads. The characteristic constant for this pendulum and its moment of inertia will be as follows:

$$
\begin{aligned}
& b=\frac{m g A^{2}}{4 l} \\
& I=\frac{T^{2} m g A^{2}}{16 \pi^{2} l}
\end{aligned}
$$

Where:

$A$ is the distance between the vertical threads;

$l$ is the length of the vertical threads.

In this case the equations (25) and (26) might be expanded to the following form:

$$
I_{V}=I_{S}+I_{E}+I_{A}=\frac{T^{2} m g A^{2}}{16 \pi^{2} l} .
$$

\subsubsection{Descending Weight Method}

This method is useful for determining the moments of inertia of rotational bodies, and the aircraft do not belong to this variety. For this reason we do not include it as a possible method for experimental determination of the moments of inertia of joined-wing UAV.

\subsubsection{Choice of a Method for the Experimental}

Determination of Mass Moments of Inertia of A Joined-Wing UAV

From the above-mentioned methods we consider the most appropriate method for the experimental determination of mass moments of inertia of a joined-wing UAV to be the physical pendulum, due to the small oscillation velocity, which diminishes the errors caused by the air resistance.

\subsubsection{Determination of Mass Moments of Inertia of a} Joined-Wing UAV by Flight Tests.

With functioning test models available, the moments of inertia might be determined with flight tests, by applying standard deflections of control planes and by measuring the resulting 
angle velocities and accelerations about the different axis, the moments of inertia can be calculated. Because of the fact that these measurements will be subjected to interference from environmental factors that must be accounted for, we shall leave this for our future work and out of the scope of the current article.

\section{EXPERIMENTAL RESULTS}

\subsection{Experimental Stand for the determination of}

\section{Mass Moments of Inertia of A Joined-Wing UAV}

In the UAV laboratory of Technical University-Sofia, Branch Plovdiv is setup an experimental stand for determination of moments of inertia fig. 3. The physical pendulum method is used [8]. With the addition of supplementary pivots the torsion pendulum method is applied.

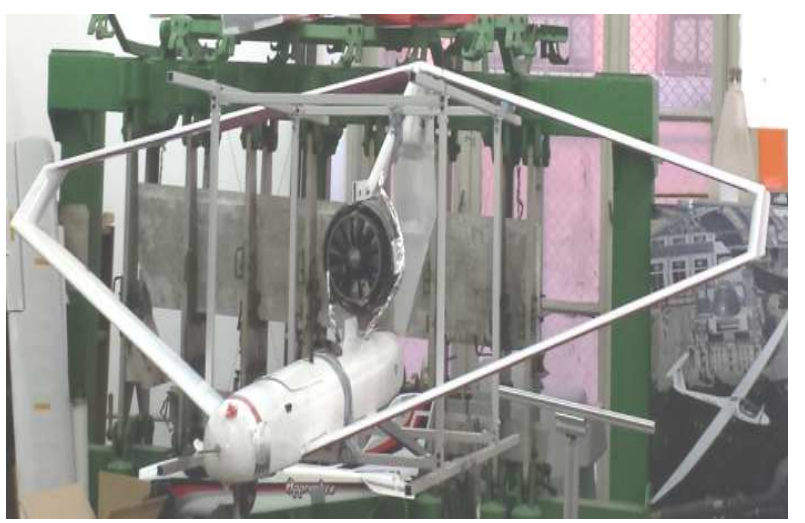

Fig 3: .Experimental determination of the moments of inertia

For the determination of the moments of inertia about the body-axis coordinate system three series of measurements were performed applying the physical pendulum method. The time for 50 oscillation cycles (100 passes through equilibrium point) and the mean value was calculated. The mass of the UAV with the cradle is $7.480 \mathrm{~kg}$., and that of the cradle alone $0.504 \mathrm{~kg}$.

\subsubsection{Measurement of Ixx}

The measurement of the moment of inertia about the $\boldsymbol{X}$ axis is performed with the fixture shown on fig. 4. The measured moment of inertia in this case is $0.384 \mathrm{~kg} \cdot \mathrm{m}^{2}$. The distance from the pivot axis to the center of mass of the UAV-cradle combination is $2.36 \mathrm{~m}$. The measured time for one oscillation is $3,243 \mathrm{~s}$. The calculated moment of inertia $\boldsymbol{I}_{x x}$ of the UAV is $1.496 \mathrm{~kg} \cdot \mathrm{m}^{2}$.

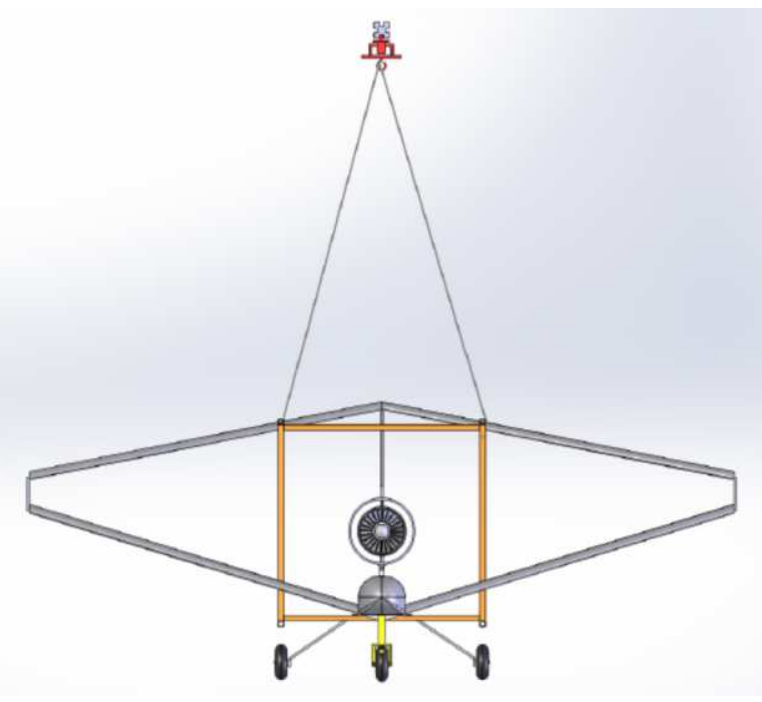

Fig. 4. Experimental fixture for the measurement of $\boldsymbol{I}_{\boldsymbol{x}}$

\subsubsection{Measurement of $I_{y y}$}

The measurement of the moment of inertia about the $\boldsymbol{Y}$ axis is performed with the fixture shown on fig. 5. The measured moment of inertia in this case is $0.384 \mathrm{~kg} \cdot \mathrm{m}^{2}$. The measured moment of inertia in this case is $0.384 \mathrm{~kg} . \mathrm{m}^{2}$. The distance from the pivot axis to the center of mass of the UAV-cradle combination is $2.36 \mathrm{~m}$. The measured time for one oscillation is $3,223 \mathrm{~s}$. The calculated moment of inertia $\boldsymbol{I}_{\boldsymbol{y} \boldsymbol{y}}$ of the UAV1, $473 \mathrm{~kg} \cdot \mathrm{m}^{2}$.

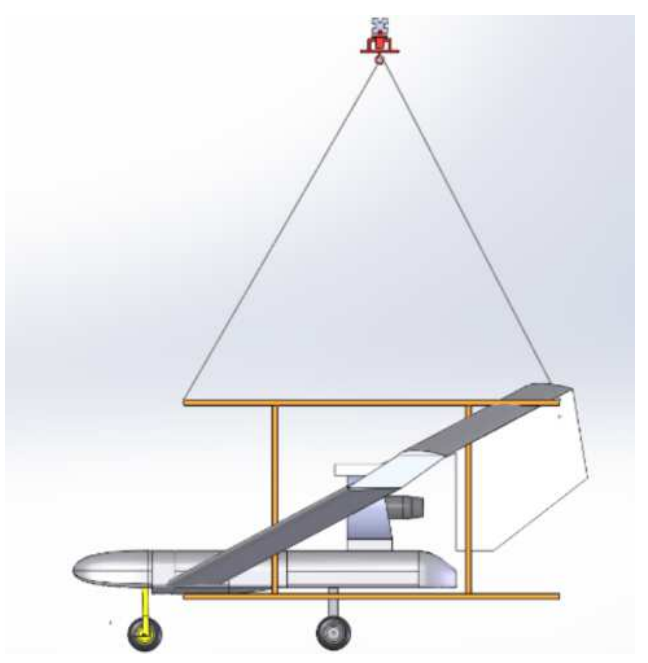

Fig. 5.Experimental fixture for the measurement of $\boldsymbol{I}_{\boldsymbol{y} y}$

\subsubsection{Measurement of $I_{z z}$}

The measurement of the moment of inertia about the $\boldsymbol{Z}$ axis is performed with the fixture shown on fig. 6.The measured moment of inertia in this case is $0.396 \mathrm{~kg} . \mathrm{m}^{2}$. The distance 
from the pivot axis to the center of mass of the UAV-cradle combination is $2.48 \mathrm{~m}$ The measured time for one oscillation is $3,263 \mathrm{~s}$. The calculated moment of inertia $\boldsymbol{I}_{z z}$ of the UAV e $1.486 \mathrm{~kg} \cdot \mathrm{m}^{2}$.

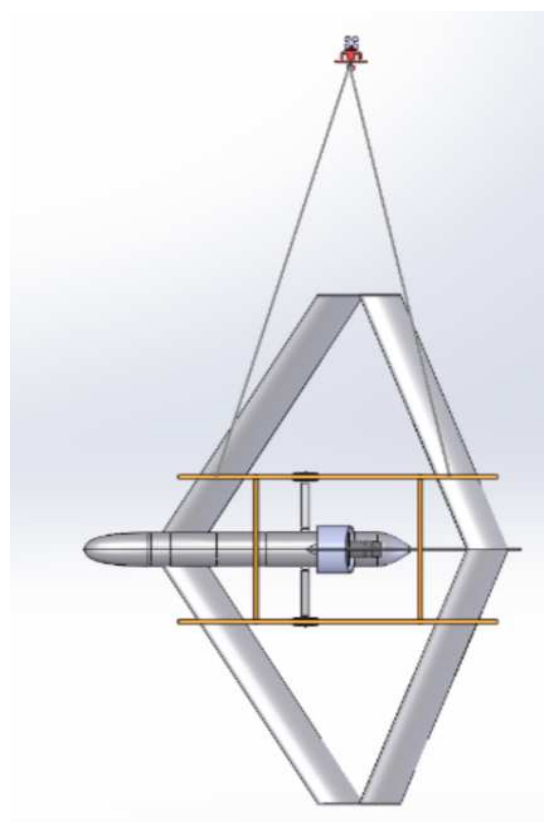

Fig. 6. Experimental fixture for the measurement of $I_{z z}$

\section{METHODOLOGY FOR THE DETERMINATION OF MASS MOMENTS OF INERTIA OF A JOINED WING UAV}

On the ground of the investigations described in this paper, a methodology for the determination of mass moments of inertia of a joined-wing UAV for each stage of design is created. The methodology applies the following algorithm.

At the conceptual design stage for each variant the moments of inertia are approximately estimated using equation (). The results are applied for the base variants election through the means of optimization and analysis of basic characteristics.

At the preliminary design stage an initial 3D model of the UAV is generated, with the help of which more precise moments of inertia are calculated.

At the detailed design stage, experimentally, applying the physical or torsion pendulum methods, the moments of inertia of the various elements of UAV are measured and their 3D models are corrected using the experimental results. The complete 3D model created using contemporary CAD/CAM software provides with sufficient accuracy the values of the moments of inertia of a joined-wing UAV.
After the construction of test articles applying the physical pendulum method the moments of inertia are measured and if necessary, the results from the previous stages of design are corrected.

\section{CONCLUSIONS}

An analytical model for the approximate calculation of moments of inertia at the conceptual design stage is proposed. The model might be applied for the comparative evaluation of the variants under review.

A complete 3D model of a joined-wing UAV is constructed and results of higher fidelity for the values of moments of inertia than the analytical model are obtained.

A stand for the experimental determination of moments of inertia of UAV is developed and constructed. A test model of a joined-wing UAV is constructed and its moments of inertia are experimentally determined.

Moments of inertia $\boldsymbol{I}_{x x}$ and $\boldsymbol{I}_{z z}$ of a joined-wing aircraft will be several times less than those of an equivalent aircraft utilizing a more conventional configuration as normal, flying wing or canard. This is due to the fact that with the joined-wing the span and mass are smaller. As a result smaller roll and direction control surfaces will be needed and the required power of the control mechanisms will be less, than the ones needed to achieve comparative performance with aircraft of other configurations.

The moment of inertia $\boldsymbol{I}_{\boldsymbol{y} \boldsymbol{y}}$ of a joined-wing aircraft will be greater than the equivalent moment of analogous aircraft because of the big distance between the centers of mass of the front and rear wings to the center of mass of the aircraft The bigger pitch control surfaces and the possibility for direct lift control, that can be realized with the joined-wing configuration might provide equivalent or even better performance than that of equivalent aircraft of other configurations.

\section{FUTUREWORK}

As a future study the moments of inertia of a joined-wing UAV will be determined with flight tests. The results that will be obtained will be analyzed and compared with the ones delivered by the described in part 6methodology, in order to evaluate its fidelity.

\section{ACKNOWLEDGEMENTS}

The authors would like to thank the Technical University of Sofia and the Bulgarian Industrial Group AD for their financial support for the realization of the experiments. 


\section{REFERENCES}

[1] Panajotov H., D. Zafirov, An Aerodynamic Optimization of Joined-Wing for Unmanned Aerial Vehicle, Journal of the Technical University-Sofia, Plovdiv branch, Vol. 13(8), 2006, pp. 12-19.

[2] Panajotov H., P. Stoyanov, Calculation of Joined-Wing Aerodynamic Center Location, Journal of the Technical University-Sofia, Plovdiv branch, Vol. 14(2), 2009, pp. 387-392.

[3] Panajotov H., Design Parameters Optimization of Joined Wing UAV, International Conference Trans\& Motauto'07 Proceedings,08-10 November 2007, pp. 103-106

[4] Panajotov H., J. Hadjiev, Investigation of Aerodynamics of UAV for Autonomous Flight Realization, International Conference BulTrans-2011 Proceedings, 27-30September 2011, pp. 103-106

[5] Panayotov H., Investigation of DutchRoll of a JoinedWing Aircraft, Proceedings of Scientific Conference on Aeronautics, Automotive and Railway Engineering and Technologies BulTrans-2012, 26-28 September 2012, Sozopol, Bulgaria, pp. 84-87

[6] Kuprikov M., Dolgov O. Especially revealing momentinertial image advanced aircraft in the early stages of design. Bulletin of the MAI.V.17, № 2, Aeronautical engineering, Moscow, 2010

[7] Stengl, Robert. Flight Dynamics.Princet on University Press, Oxford, Princet on, NJ, 2004

[8] Halder, A. Agarwal V., R. Garhwal, and M. Sinha, "Determination of InertialCharacteristics of a HighWingUnmannedAirVehicle", Journal of Institute of Engineers, India, Vol. 89, 2008

\section{BIOGRAPHIES}

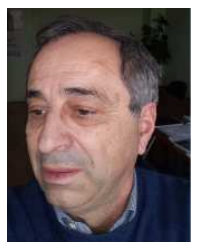

Assoc. prof. DimoZafirovPhD is lecturer in design of aircraft and has professional experience in the field of UAVs. His interests are focused on the study of aircraft with joined wing.

\section{NOMENCLATURE}

$\boldsymbol{O}_{\boldsymbol{x y z}}$-body-axis coordinate system with an origin $\boldsymbol{O}$ at the center of mass of the aircraft; $\boldsymbol{r}$-radius vector of a body point; $\boldsymbol{\rho}(\boldsymbol{r})$-density of the body at a point;

$\boldsymbol{d}(\boldsymbol{r})$-distance of the point to the axis;

$\mathbf{d} \boldsymbol{V}(\boldsymbol{r})$-elementary volume at a point;

$\boldsymbol{b}$-characteristic constant, depending on the type, size and weight of a pendulum;

$\boldsymbol{\theta}$-angular displacement of the pendulum;

$\ddot{\boldsymbol{\varphi}}-$ Angular acceleration of body around the axis $x$;

$\ddot{\boldsymbol{\theta}}$-Angular acceleration of body around the axisy;

$\ddot{\boldsymbol{\psi}}$-Angular acceleration of body around the axisz; $\overrightarrow{\boldsymbol{\alpha}}$ - angular velocity vector of the body;

$\boldsymbol{g}$ - earth gravity acceleration;

I-matrix of moment of inertia of body;

$\boldsymbol{I}_{\boldsymbol{S}}$-mass moment of inertia of the structure of the measured body;

$\boldsymbol{I}_{\boldsymbol{E}}$-mass moment of inertia of the air, entrapped in the measured body;

$I_{A}$-mass moment of inertia of the air, dragged by the body during the experiment;

$\boldsymbol{I}_{G}$-mass moment of inertia of the cradle of the stand for measuring inertial moments;

$I_{\text {C.G.. }}$-mass moment of inertia of a body about axis of oscillation crossing through its center of mass;

$I_{x x}$-mass moment of inertia of a body about axis of oscillation $x$ crossing through its center of mass;

$\boldsymbol{I}_{\boldsymbol{x} \boldsymbol{x}_{j}}$-moment of inertia of the $\boldsymbol{j}_{\text {-th }}$ element of aircraft aboutxaxis;

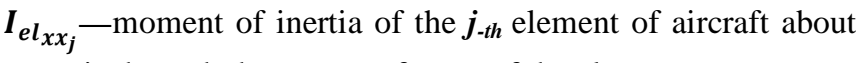
$\boldsymbol{x}_{\boldsymbol{j}}$, axis through the center of mass of the element;

$\boldsymbol{I}_{y y}$-mass moment of inertia of a body about axis of oscillation $\boldsymbol{y}$ crossing through its center of mass;

$\boldsymbol{I}_{z \mathbf{z}}$-mass moment of inertia of a body about axis of oscillation $z$ crossing through its center of mass;

$\boldsymbol{m}_{\boldsymbol{J}}$ - mass of the $\boldsymbol{j}_{-\boldsymbol{t} \boldsymbol{h}}$ element;

$\boldsymbol{r}_{\boldsymbol{J}}$ - distance from the centre of mass of the $\boldsymbol{j}_{-\boldsymbol{t} h}$ element of aircraft to $x$ axis;

$\boldsymbol{l}$ - distance from the axis of oscillation to $\boldsymbol{x a x i s}$ of the bodycradle system;

$\boldsymbol{l}^{\prime}$ - distance from the axis of oscillation to $\boldsymbol{x a x i s}$ of the bodycradle system during the $\boldsymbol{i}$-th experiment, $\boldsymbol{i}=1,2,3$;

$\boldsymbol{m}$ - mass of body;

$\boldsymbol{m}_{A^{-}}$adjoined mass of the air, dragged by the body;

$\boldsymbol{T}$ - period of oscillation of the body-cradle system;

$\boldsymbol{V}$ - body volume, filled with air;

$\boldsymbol{\rho}-$ density of the air during the experiment;

W - weight of the aircraft;

$\boldsymbol{W}_{\boldsymbol{G}}$ - weight of the cradle;

UAV - unmanned air vehicle; 